\title{
Recognition of Indian Sign Languages
}

\author{
Sahara Shetty ${ }^{1}$, and Yashaswi G. $\mathbf{P}^{2}$ \\ ${ }^{1,2}$ Students, Department of Computer Science and Engineering, Srinivas Institute of Technology, Mangalore, India \\ Correspondence should be addressed to Sahara Shetty; saharashetty1999@gmail.com
}

Copyright ( 2021 Made Sahara Shetty et al. This is an open access article distributed under the Creative Commons Attribution License, which permits unrestricted use, distribution, and reproduction in any medium, provided the original work is properly cited.

\begin{abstract}
The focal point of this project is about developing a user interface to aid people who have hearing and speaking disabilities. This application would help in recognizing the different signs with respect to the single user. The real-time image is captured using a webcam and dynamically stored. The elements are initialized and saved in KN Neighbor classifiers. These classifiers are used to load the KN Neighbor model. This model predicts the signs dynamically and is built using machine learning techniques. Mobile Net is used as a machine learning package. This model was implemented in three phases. The first phase deals with the user interface where user images are captured using a webcam. In the second phase, the initialized elements are used by the KN Neighbor classifier and then stored as the KN Neighbor model. The third phase involves the prediction of signs.
\end{abstract}

KEYWORDS- Indian sign language, Tensor flow, squeeze net

\section{INTRODUCTION}

There are a lot of deaf and dumb people around the world. Deaf is the disability that makes people unable to hear and dumb is a disability which makes people unable to speak. These deaf or dumb people can do a lot of other works like normal people the only thing which makes them different from the others is the difficulty they face in communication. The way for these deaf or dumb people to communicate with normal people and themselves is through sign language . Recognition of sign language is based on hand gestures and recognizing these sign languages is a problem that is faced by lot of people. More than sixteen lakh people in India have speech disorder which makes them difficult to communicate. Many experts in India believe that there is a shortage of special schools for these deaf and dumb people. Only five percentage of these people attend these schools. The hearing impaired people are ignored from the society because the normal people never try to learn Indian sign Languages and they don't try to interact with these hearing impaired people. This will lead to very poor literacy among these people. These deaf or mute people communicate through sign language which is difficult for the normal ones to understand. Hence we have come up with a project which would help in recognition of sign language in which the camera captures the signs or hand gestures and output is given in form of texts which is easily understandable. Clear understanding about the interface to the users is done using manual entries of sign language. The objective of this paper is to communicate with speech and hearing-impaired people. There is a need of this system which recognises different signs and convey them to normal people. This system helps to communicate between deaf or mute people and the normal people which is important for the society. The algorithm helps in classifying the sign languages using web camera. The algorithm then categories the uploaded images. We train the algorithm by feeding sign images. In this model we train it using 30 gestures. The algorithm then detects the sign shown by individual. Depending on training of all gesture samples the system provides output accuracy.

\section{RELATED WORK}

People communicate with each other to covey their thoughts, ideas and experiences. But there are few people around the world like deaf and dumb who cannot convey their messages like others. In the previous works,[1] the idea proposed is smart glove which converts sign language to speech output. Communication for speech impaired person is produced because the glove facilitates in manufacturing artificial speech. Since head and gesture express as soon as reaction of user views it plays a vital role compared to other gestures like body, face. Basically, this is a microcontroller and data glove-based system. Microcontroller based system converts specified movements into human understandable voice and data glove can detect all the movement of hands. Another work,[2] shows a system which connects the dumb people to others. In this, a webcam is placed where the physically impaired person would place his finger ahead of the camera and then the web camera captures the hand gestures, perform image processing using the principal component analysis algorithm. The captured co-ordinates will be mapped with the previously stored and accordingly pictures will be identified from the database. This way the speech impaired person can communicate. This way the sentences later will be translated into audio speech. Another work [3], where a method is planned which will produce the employment of hand gestures for Indian linguistic communication is recognized. In [4], artificial mouth for speaking is proposed where expressions and hand motion are used by impaired to communicate. This system is mainly based on monitoring sensors which would help the people to convey their thoughts. In work [5] messages, ideas are conveyed through hand gestures. Using the concept of thresholding numbers from 0 to 10 are recognized which consist of three steps. At the beginning images are captured after applying thresholds numbers are 
recognized. To complete these steps the person must be aware about using the gloves.

\section{SYSTEM IMPLEMENTATION}

The system architecture of Indian Sign Language recognition is designed in such a way that one can provide custom made or also trained standard sign gestures i.e., ASL, ISL, etc. The datasets are trained using squeeze.net library with Tensor Flow. Using KNN classifier the trained datasets are classified and predicted. The predicted or recognized output is arranged as a query of texts. The flowchart states that user has to start the server and load the web interface. For query delimiter purpose the user should provide start and stop sign. In the training mode, the user can provide custom gestures except for standard ones and later he can enter into prediction mode where the trained model will predict the gestures and build an text query. If the predicted output doesn't attain accuracy, model can be retrained with more sets of sign gestures.

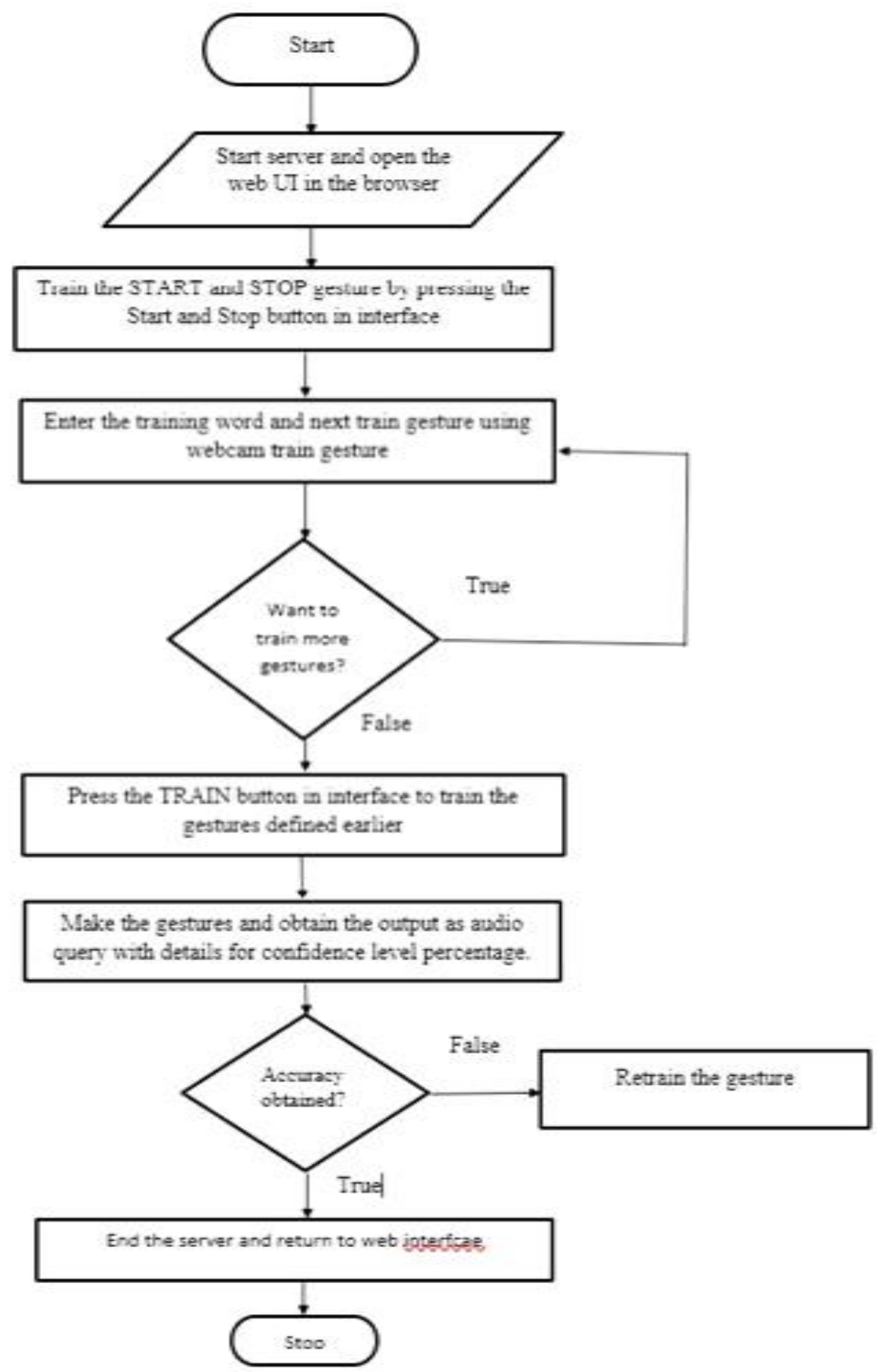

Fig. 1: System Implementation 


\section{EXPERIMENTAL RESULT}

The Table given below represents the result analysis table where it has some training dataset which consist of any number of gesture images. In non-real time the datasets consist of 10 gesture images with dimensions $277 * 277$ webcam capture window size in 10 different angles. It has horizontal and vertical resolution of 96dpi. In real time the datasets consist of gestures of different sign language with dimensions $277 * 277$. The horizontal and vertical resolution is 96dpi. The result analysis table contains keywords such as where TP indicates number of true positives, TN indicates number of true negatives, FP indicates number of false positives and FN indicates false negative results.

Table 1: Result Analysis Table

\begin{tabular}{|l|c|l|l|l|l|l|}
\hline Gesture Name & $\begin{array}{c}\text { No. of Testing } \\
\text { Images }\end{array}$ & \multicolumn{1}{|c|}{ TP } & TN & FP & FN & Result \\
\hline Start & 30 & 29 & 1 & 0 & 0 & 0.91 \\
\hline Stop & 30 & 29 & 1 & 0 & 0 & 0.91 \\
\hline Ok Google & 10 & 9 & 1 & 0 & 0 & 0.91 \\
\hline Good Morning & 10 & 8 & 2 & 0 & 0 & 0.82 \\
\hline What & 10 & 9 & 1 & 0 & 0 & 0.91 \\
\hline Time & 10 & 9 & 1 & 0 & 0 & 0.91 \\
\hline Read & 10 & 8 & 1 & 1 & 0 & 0.81 \\
\hline News & 10 & 8 & 1 & 1 & 0 & 0.81 \\
\hline Light & 10 & 9 & 1 & 0 & 0 & 0.91 \\
\hline ON & 10 & 8 & 1 & 0 & 1 & 0.81 \\
\hline
\end{tabular}

Average overall accuracy $=0.871=87.1 \%$

\section{CONCLUSION AND FUTURE SCOPE}

The problem of deaf and dumb candidates is supported by, being bridged by creating a web application. This web application allows the users to interact with the people of real world with the sign language gestures. It allows the user to train their custom made as well as standard sign language gestures to interact with the normal user to do the day-to-day tasks. It connects the gap between the mutual communication problems caused due to lack of knowledge of sign languages to normal users. This web app results in efficient time usage. The system helps to improve the performance. Maintaining the project is easy and manageable. It is easily understandable by the user. In future the web application can be converted into android applications. It can be modified in such a way that application can be used by organizations other than educational institutions. Organizations can add functionalities depending on their needs.

\section{CONFLICTS OF INTEREST}

The authors declare that they have no conflicts of interest.

\section{REFERENCES}

[1] Abhishek Jain, Lakshita Jain, Ishaan Sharma, Abhishek Chauhan, TECH, Department Of ECE, SRMIST,“ Image Processing Based Speaking System For Mute People Using
Hand Gestures", International Journal Of Engineering Sciences \& Research Technology, 2018.

[2] Ashish G. Bairagi, Y.D. Kapse P.G. Student, Department of E\&TC Engineering, GCOEJ, Jalgaon, Maharashtra, "Survey on Sign language to Speech Conversion", International Journal of Innovative Research in Computer \& Communication Engineering, 2018.

[3] Aruljothy, Arunkumar, Ajitraj, Yayad Damodran, Jeevanantham, Dr. M. Subba, UG Scholar, Department of Instrumentation and Control Engineering, Sri Manakula Vinayagar Engineering College, Puducherry, "Hand Gesture Recognition Using Image Processing for Visually Impaired \& Dumb Person", International Journal of Advanced Research in Computer and Communication Engineering ISO, 2014.

[4] V. Padmanabhan, M. Sornalatha, "Hand gesture recognition and voice conversion system for dumb people", International Journal of Scientific \& Engineering Research, 2014.

[5] Bhavsar Swapna1, Futane Pravin1 and V. Dharaskar Rajiv, Sinhagad college of engineering, Pune University, "Hand Gesture Recognition System for Numbers Using Thresholding", Research Center Amravati University Amravati, India, 2018.

\section{ABOUT THE AUTHORS}

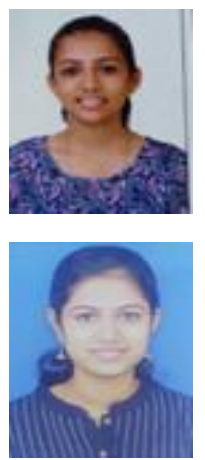

Sahara Shetty, Under Graduate Final Year Student, Department of Computer Science and Engineering, Srinivas Institute of Technology, Valachil, Managlore, Karnataka, India

Yashaswi G.P, Under Graduate Final Year Student, Department of Computer Science and Engineering, Srinivas Institute of Technology, Valachil, Managlore, Karnataka, India 